\title{
A total-population multigenerational family clustering study of autoimmune diseases in obsessive-compulsive disorder and Tourette's/chronic tic disorders
}

\author{
David Mataix-Cols, $\mathrm{PhD}^{1,2, \S}$, Emma Frans, $\mathrm{PhD}^{3, \S}$, Ana Pérez-Vigil, $\mathbf{M D}^{1}$, Ralf Kuja-Halkola, \\ $\mathrm{PhD}^{3}$, Caroline Gromark, $\mathbf{M D}^{1,2}$, Kayoko Isomura, $\mathbf{M D}, \mathrm{PhD}^{1,2}$, Lorena Fernández de la Cruz, \\ $\mathbf{P h D}^{1}$, Eva Serlachius, MD, PhD ${ }^{1,2}$, James F. Leckman, MD, $\mathrm{PhD}^{4}$, James J. Crowley, $\mathrm{PhD}^{1,5}$, \\ Christian Rück, MD, PhD ${ }^{1,2}$, Catarina Almqvist, MD, PhD $^{3,6}$, Paul Lichtenstein, PhD $^{3}$, and \\ Henrik Larsson, $\mathrm{PhD}^{3,7}$ \\ ${ }^{1}$ Centre for Psychiatry Research, Department of Clinical Neuroscience, Karolinska Institutet, \\ Stockholm, Sweden \\ ${ }^{2}$ Stockholm Health Care Services, Stockholm County Council, Stockholm, Sweden \\ ${ }^{3}$ Department of Medical Epidemiology and Biostatistics, Karolinska Institutet, Stockholm, Sweden \\ ${ }^{4}$ Child Study Center, Yale University School of Medicine, New Haven, Connecticut, USA \\ ${ }^{5}$ Departments of Genetics and Psychiatry, University of North Carolina at Chapel Hill, USA \\ ${ }^{6}$ Pediatric Allergy and Pulmonology Unit, Astrid Lindgren Children's Hospital, Karolinska \\ University Hospital, Stockholm, Sweden \\ ${ }^{7}$ School of Medical Sciences, Örebro University, Örebro, Sweden
}

\section{Abstract}

The association between obsessive-compulsive disorder (OCD) and Tourette's/chronic tic disorders (TD/CTD) with autoimmune diseases (AD) is uncertain. In this nationwide study, we sought to clarify the patterns of comorbidity and familial clustering of a broad range of $\mathrm{AD}$ in individuals with OCD and/or TD/CTD and their biological relatives. From a birth cohort of 7,465,455 individuals born in Sweden between 1940 and 2007, we identified 30,082 OCD and 7,279 TD/CTD cases in the National Patient Register and followed them up to December 31, 2013. The risk of $40 \mathrm{AD}$ was evaluated in individuals with OCD and/or TD/CTD and their first (siblings, mothers, fathers), second (half siblings), and third degree (cousins) relatives, compared to population controls. Individuals with $\mathrm{OCD}$ and TD/CTD had increased comorbidity with any AD (43\% and 36\%, respectively) and many individual AD. The risk of any AD and several individual $\mathrm{AD}$ was consistently higher among first-degree relatives than among second- and third-degree relatives of $\mathrm{OCD}$ and TD/CTD probands. The risk of AD was very similar in mothers, fathers, and siblings of OCD probands, whereas it tended to be higher in mothers and fathers of TD/CTD probands (compared to siblings). The results suggest a familial link between AD in general (i.e.,

Correspondence to: Professor Mataix-Cols, Karolinska Institutet, Department of Clinical Neuroscience; Child and Adolescent Psychiatry Research Center, Gävlegatan 22 (Entré B), floor 8; SE-11330 Stockholm, Sweden. david.mataix.cols@ki.se.

\$oint first authors 
not limited to streptococcus-related conditions) and both OCD and TD/CTD. Additional motherspecific factors, such as the placental transmission of antibodies, cannot be fully ruled out, particularly in TD/CTD.

\section{Introduction}

Immunological factors are increasingly recognized as etiologically important in a range of neuropsychiatric disorders, such as autism spectrum disorder, ${ }^{1}$ schizophrenia, ${ }^{2}$ or bipolar disorder. ${ }^{3}$ The potential link between immunological factors and psychiatric symptoms has been much debated in obsessive-compulsive disorder (OCD) and Tourette's/chronic tic disorders (TD/CTD), due to the still controversial link between group A streptococcus infections and the abrupt onset of these psychiatric symptoms in a small group of children. 4,5

One way to determine if immunological factors are involved in the causation of OCD and/or $\mathrm{TD} / \mathrm{CTD}$ is to examine if there is increased comorbidity and family history of autoimmune diseases $(\mathrm{AD})$ in these psychiatric patients. Because $\mathrm{AD}$ share some of the same risk factors (e.g., cytokine dysfunction, genetic and environmental factors), ${ }^{6-9}$ tend to coexist in the same individuals, and co-aggregate in the same families, ${ }^{10-12}$ finding an above-chance prevalence of $\mathrm{AD}$ in patients with $\mathrm{OCD}$ and/or TD/CTD and their relatives would support a possible role of the immune system in the etiology of these disorders. ${ }^{13}$

While the association between rheumatic fever - particularly its neurological manifestation, Sydenham's chorea -, OCD, and TD/CTD has been well established, the link with other classic $\mathrm{AD}$ is far less clear. ${ }^{13-16}$ The previous literature has been scarce and severely limited by methodological issues, such as the study of small sample sizes mainly recruited from specialist clinics, the use of cross-sectional designs, lack of blind assessors, lack of control groups, and the use of self-report measures known to have poor psychometric properties. ${ }^{13}$

The strongest evidence of the association between $\mathrm{AD}$ and $\mathrm{OCD}$ or TD/CTD originates from a handful of family studies suggesting that the mothers of individuals with these disorders have elevated rates of AD. In a specialty clinic-based study, ${ }^{17}$ the mothers of 107 children and adolescents with OCD and/or TD/CTD were interviewed regarding their history of AD. Nearly $18 \%$ of the mothers reported at least one AD; this figure was higher for the mothers of children fulfilling likely criteria for PANDAS (Pediatric Autoimmune Neuropsychiatric Disorder Associated with Streptococcal Infection) ( $\mathrm{n}=40 ; 25 \%)$, compared to the mothers of children with unlikely PANDAS ( $\mathrm{n}=67 ; 13.4 \%)$. A longitudinal population-based study from Denmark examined the risk of TD $(n=2,442)$ in the offspring of mothers with 31 different AD. A maternal history of $\mathrm{AD}$ was found in 110 cases, corresponding to an increased risk of TD of approximately $22 \%$. The most common maternal AD were ulcerative colitis, rheumatoid arthritis, thyrotoxicosis, and multiple sclerosis, although power was limited to examine the risk for many of the individual AD. ${ }^{18}$ In two case-control family studies that analyzed the same Brazilian sample using different statistical approaches, ${ }^{19,}{ }^{20}$ the rate of OCD and related disorders, including tic disorders, was significantly higher among firstdegree relatives of individuals with rheumatic fever than among first-degree relatives of controls (14.7\% vs $7.3 \%),{ }^{19}$ and the risk of OCD and related disorders was increased by the 
presence of either rheumatic fever or Sydenham's chorea in another family member. ${ }^{20}$ Conversely, another family study ${ }^{21}$ found no significant differences in the rates of OCD in 72 mothers of children and adolescents with inflammatory bowel disease, compared to 44 mothers of children and adolescents with cystic fibrosis (a non-autoimmune genetic disease).

Taken together, the results of these family studies support the hypothesis that immunological factors may play a role in the etiology of at least some individuals with OCD and/or TD/ CTD, but these findings require replication in large population-based samples. Furthermore, the mechanisms of such association, if confirmed, are yet to be established. One possibility is that $\mathrm{OCD}$ and TD/CTD share genetic risk factors with $\mathrm{AD}$. If the shared genetic risk hypothesis were correct, we would expect that the link between $\mathrm{AD}$ and these neuropsychiatric syndromes is not limited to mothers but also other first-degree relatives. Further, we would expect that the risks decrease with increasing genetic distance (for example, the risks for first-degree relatives should be higher than those for second- and third-degree relatives). An alternative - and perhaps not mutually exclusive - hypothesis is that mothers transmit antibodies to their offspring via the placenta. ${ }^{22,23}$ If the maternal transmission hypothesis were correct, we would expect that the risk for mothers would be significantly higher than the risk for fathers and other first-degree relatives.

In this study, we analyzed data from a large birth cohort of OCD and TD/CTD cases derived from the Swedish population-based registers to address the following questions: 1) are patients with $\mathrm{OCD}$ and/or TD/CTD more likely to have comorbid $\mathrm{AD}$, compared to population controls?; 2) are biological relatives of patients with OCD and/or TD/CTD more likely to have $\mathrm{AD}$, compared to relatives of unaffected individuals?; 3) does the familial risk decrease with increasing genetic distance?; and 4) do mothers have higher risks than fathers or other first-degree relatives?

\section{Methods}

\section{Study design}

We conducted a population-based birth cohort study to examine the comorbidity and familial clustering between $\mathrm{AD}$ and $\mathrm{OCD}$ and between $\mathrm{AD}$ and TD/CTD. We linked information from various Swedish national registers using the unique personal identification number, a national registration number assigned to each Swedish citizen at birth or immigration. ${ }^{24}$ These national registration numbers are randomly replaced with unique sequence numbers when register data is used for research purposes.

The Stockholm Regional Ethical Review Board approved the study (2013/862-31/5). The requirement for informed consent was waived because the study was register-based and the included individuals were not identifiable at any time.

\section{Study cohort}

We selected a cohort of individuals born between 1940 and 2007 and followed them up until December 31, 2013. We excluded individuals who died or emigrated before the age of 10 years. Information about migration and death was derived from the Total Population Register and the Cause of Death Register. 
Individuals with OCD and TD/CTD were identified in the National Patient Register (NPR) using previously validated algorithms. ${ }^{25}$ The NPR includes psychiatric inpatient discharge diagnoses in Sweden since 1973, recorded according to the International Classification of Diseases (ICD) 8th, 9th, and 10th editions. ${ }^{26-29}$ Since 2001, the register also includes information about psychiatric outpatient care.

The outcomes were diagnoses of 40 widely accepted $\mathrm{AD}^{30}$ in the index person and in relatives of an index person identified in the NPR (listed in Supplementary Table 1, together with their corresponding ICD codes). Additional analyses were conducted on specific AD that were most prevalent in the NPR and had sufficient number of observations for analysis (>10 affected individuals). Many of the ICD codes for individual AD in the NPR have been subject of validation studies and found to have high validity. $29,32,33$

Information about relatives was derived from the Multi-Generation Register, which contains information about biological parents of an index person. A prerequisite for being included in the register is that the index person was born after January 1, 1932 and ever registered as living in Sweden after 1960. For immigrants to Sweden, similar information exists for those who became citizens before 18 years of age together with one or both parents. The biological father of the offspring is assumed to be the husband of the mother at the time of birth or identified "by acknowledgment" for unwed mothers. ${ }^{24}$

\section{Statistical analyses}

Data managing and analyses were conducted in SAS statistical software version 9.4 (SAS Institute, Inc) and Stata IC/14.1. We estimated odds ratios (OR) to quantify the association between OCD and TD/CTD and AD with logistic regression analyses. All statistical tests employed two-sided 5\% level of significance and associated two-sided 95\% Wald-type confidence intervals (CI) were calculated. We employed the robust variance estimator (sandwich estimator) to handle clustering of data.

Individual-level analyses-First, we estimated the risk of any AD in individuals with a diagnosis of OCD, and separately in individuals with a diagnosis of TD/CTD. Individuals born between 1940 and 2007 with a lifetime diagnosis of OCD or TD/CTD were considered exposed and those without these disorders were considered unexposed. We also examined the risk of individual $\mathrm{AD}$ in patients with $\mathrm{OCD}$ and TD/CTD (those with $>10$ observations). All analyses adjusted for birth year and gender.

Family-level analyses-We first linked information about biological parents to our cohort and analyzed the risk of any $\mathrm{AD}$ (and also specific $\mathrm{AD}$ ) in parents of individuals affected with OCD and TD/CTD compared to parents of unaffected individuals. For the sibling and cousin analyses we used the same birth cohort as when studying the individual risk (born between 1940 and 2007). Full siblings were defined as individuals who share two biological parents and half siblings were defined as individuals who share one parent. Cousin analyses only included full cousins, defined as having a common grandfather and grandmother. All analyses adjusted for birth year and gender of the index person as well as for birth year of the parent/sibling/cousin. 
Sensitivity analyses-In order to ensure that we studied the independent transmission of these conditions, all family analyses were repeated excluding OCD and TD/CTD probands with diagnosed $\mathrm{AD}$ as well as relatives with either $\mathrm{OCD}$ or TD/CTD.

Additionally, individual-level and family-level analyses for any AD were repeated on a subgroup of individuals born between 1963-1983 to ensure complete follow-up from age 10 to at least age 30, thus avoiding issues with left truncation (i.e., exposure and/or outcome happening before the register started and thus missing) and right censoring (i.e., study ending when individuals were still young).

We also repeated the individual- and family-level analyses for any AD excluding individuals diagnosed before 2001, date from which outpatients were first included in the register, to ensure that more severe patients (i.e., inpatients) were not biasing the results.

\section{Results \\ Study cohort}

The cohort included a total of 7,465,455 unique individuals of which 30,082 had a diagnosis of OCD and 7,279 had a diagnosis of TD/CTD. Moreover, our cohort included 7,416,593 individuals with information about both biological parents, 10,670,459 full sibling pairs, 1,420,623 maternal half-sibling pairs, 1,841,737 paternal half-sibling pairs, and 25,856,102 cousin pairs.

\section{Individual-level analysis}

Individuals with $\mathrm{OCD}$ had a $43 \%$ increased risk of any $\mathrm{AD}$, compared to individuals without $\mathrm{OCD}(\mathrm{OR}=1.43,95 \% \mathrm{CI}=1.37-1.49$; Table 1$)$. In the analyses of individual $\mathrm{AD}$, the risk was significantly elevated for Sjögren's syndrome (94\% increase), celiac disease (76\%), GuillainBarré syndrome (71\%), Crohn's disease (66\%), Hashimoto's thyroiditis (59\%), type 1 diabetes mellitus (56\%), scarlet fever (52\%), idiopathic thrombocytopenic purpura (51\%), ulcerative colitis (41\%), multiple sclerosis (41\%), and psoriasis vulgaris (32\%). Other AD (e.g., systemic lupus erythematosus or acute disseminated encephalomyelitis) were also associated with OCD, but power was limited, resulting in wide CI.

Similarly, individuals with TD/CTD were $36 \%$ more likely to be affected with any AD than members of the same cohort without TD/CTD ( $\mathrm{OR}=1.36,95 \% \mathrm{CI}=1.22-1.51$; Table 1$)$. In the analyses of individual $\mathrm{AD}$, the highest risk was found for Hashimoto's thyroiditis (106\% increase), celiac disease (67\%), scarlet fever (62\%), type 1 diabetes mellitus (37\% increase), and psoriasis vulgaris (33\% increase). Other $\mathrm{AD}$, such as Chrohn's disease or vitiligo, were also associated with TD/CTD, but CI were wide due to limited statistical power.

\section{Family-level analysis}

When examining the risk of any AD in relatives of index persons with OCD, compared to relatives of persons who did not have $\mathrm{OCD}$, we found that the association was strongest and statistically significant among first-degree relatives (Table 2). Mothers, fathers, and full siblings of individuals with OCD were each significantly more likely to be affected with an AD. The risk was marginally higher among mothers (17\% increase) and siblings (16\% 
increase) than among fathers ( $8 \%$ increase), but CI overlapped. The associations between $\mathrm{OCD}$ and $\mathrm{AD}$ in second-degree relatives (maternal or paternal half siblings) or in cousins were lower than for first-degree relatives and none were statistically significant (Table 2). Family-level analyses of individual AD followed the same general pattern (Supplementary Table 2).

Individuals with TD/CTD were statistically significantly more likely to have a first-degree relative with any $\mathrm{AD}$ (Table 2). The effect was highest among mothers of individuals diagnosed with TD/CTD (40\% increased risk), followed by fathers (31\% risk) and siblings (17\% risk), but CI overlapped. Furthermore, second- and third-degree relatives of individuals with TD/CTD did not have a significantly elevated risk of AD (Table 2). Family-level analyses of individual AD followed the same general pattern (Supplementary Table 3).

In an exploratory post-hoc analysis, we examined if individuals with both OCD and TD/CTD (OCD + TD/CTD; $n=1,276)$ had similar comorbidity and familial risks of AD. The results revealed that these individuals had similar comorbidity $(\mathrm{OR}=1.48)$ to that reported in the main analyses above. The familial estimates were similar to those of $\mathrm{TD} / \mathrm{CTD}$ probands, with one exception: the risk of $\mathrm{AD}$ in siblings was somewhat higher amongst $\mathrm{OCD}+\mathrm{TD} / \mathrm{CTD}$ probands $(\mathrm{OR}=1.38)$. However, these results need to be interpreted cautiously given the small sample size and wide confidence intervals (only 99 siblings of OCD + TD/CTD probands had at least one AD; Supplementary Table 4).

\section{Sensitivity analyses}

A similar pattern of results was obtained in sensitivity analyses excluding probands with any $\mathrm{AD}$ as well as relatives with either OCD or TD/CTD (Figure 1; Supplementary Table 5) in the sub-cohort of patients born from 1963 to 1983 (Supplementary Table 6) and in the subcohort of patients diagnosed from 2001 (Supplementary Table 7).

\section{Discussion}

In this total-population, multigenerational family study we found that both OCD and TD/CTD are highly comorbid with a broad range of AD across all organ systems. Specifically, individuals with OCD were $43 \%$ more likely, and individuals with TD/CTD $36 \%$ more likely, to have any comorbid $\mathrm{AD}$, compared to unaffected individuals from the general population.

In patients with $\mathrm{OCD}$, most individual $\mathrm{AD}$ showed an increased comorbidity rate, but the association was strongest for Sjögren's syndrome (94\% increase), celiac disease (76\%), Guillain-Barré syndrome (71\%), Crohn's disease (66\%), Hashimoto's thyroiditis (59\%), type 1 diabetes mellitus (56\%), scarlet fever (52\%), idiopathic thrombocytopenic purpura (51\%), ulcerative colitis (41\%) multiple sclerosis (41\%), and psoriasis vulgaris (32\%). Similarly, in TD/CTD, many individual AD showed an association but, due to modest statistical power for some of the rarest $\mathrm{AD}$, this only reached statistical significance for Hashimoto's thyroiditis (106\% increase), celiac disease (67\%), scarlet fever (62\%), type 1 diabetes mellitus (37\%), and psoriasis vulgaris (33\%). These results expand our previous knowledge on the subject - as previous studies had been limited by a range of methodological issues ${ }^{13}$ 
and suggest that there is a clear association between both OCD and TD/CTD and AD in general, which is not limited to streptococcal-related conditions.

The family analyses revealed significantly elevated risk of $\mathrm{AD}$ in first-degree relatives of both OCD and TD/CTD patients, but not in second- or third-degree relatives. The magnitude of the results was highest for first-degree relatives of TD/CTD probands. The results were unchanged when we excluded OCD and TD/CTD probands with diagnosed AD, as well as relatives with either OCD or TD/CTD, and in various sensitivity analyses. While our design does not allow us to confidently rule out shared environmental factors, the pattern of results is compatible with a possible shared genetic etiology between OCD and TD/CTD and AD, at least in some patients. First, the association was only statistically significant amongst firstdegree relatives. Second, siblings of probands had similar (OCD) or lower (TD/CTD) risks of $\mathrm{AD}$ than mothers and fathers (they all share about $50 \%$ of the genes) despite siblings sharing more of the environment (siblings are born in the same family, during a similar period of time, and grow up in the same household). Furthermore, maternal and paternal half-siblings (both sharing 25\% of genes but paternal half-siblings sharing less environment, as $>90 \%$ of Swedish children continue living with their mothers after a divorce) ${ }^{34}$ had very similar risks of $\mathrm{AD}$. Consistent with a potential genetic mechanism, a recent study using publicly available genome-wide association (GWAS) data to explore the genetic correlations between a range of psychiatric and medical phenotypes with immunological and/or inflammatory disorders ${ }^{35}$ revealed significant positive genetic correlations (uncorrected for multiple testing \pm standard error) between OCD and celiac disease $\left(\mathrm{r}_{\mathrm{g}}=0.39 \pm 0.18\right)$ and ulcerative colitis $\left(\mathrm{r}_{\mathrm{g}}=0.29 \pm 0.09\right)$, and negative correlations with type 1 diabetes mellitus $\left(r_{g}=-0.28 \pm 0.10\right) .{ }^{35}$ It should be noted that current published GWAS for $\mathrm{OCD}^{36,37}$ and TD, ${ }^{38}$ as well as some $\mathrm{AD}$, are underpowered to detect genome-wide significant associations and much larger studies are needed. For example, it remains unclear whether OCD or TD/CTD are associated with variation in the major histocompatibility complex region on chromosome 6 , which has a major influence on risk for many AD.

Future molecular genetic studies should focus on collection of DNA from large numbers of OCD and TD/CTD cases with rich clinical and environmental risk factor information (e.g., personal and family history of $\mathrm{AD}$, documented temporality between infection and symptom onset). At least one study of this type is currently ongoing in TD/CTD. ${ }^{39,} 40$ In addition, comparison of genetic markers in patients with $v s$ without additional neurological or immunological symptoms (PANS [Pediatric Acute-onset Neuropsychiatric Syndrome]/ PANDAS) may yield mechanistic insight. Given the complexity of these psychiatric and immunological traits, well-powered analyses are key to robust findings. An excellent demonstration of this comes from a recent analysis ${ }^{41}$ of GWAS data for more than 60,000 cases and controls representing three adult psychiatric disorders: schizophrenia, major depression, and bipolar disorder. The authors searched for biological pathways enriched for psychiatric risk variants and found that multiple immune signaling pathways were among the most robustly associated, providing a mechanistically-informative link between genetic and epidemiological studies for these disorders.

Mothers, fathers, and full siblings of individuals with OCD and TD/CTD were each more likely to be affected with an $\mathrm{AD}$, compared to unaffected individuals from the general 
population. In some analyses, particularly in TD/CTD, the risk was slightly higher among mothers than among fathers and siblings, but these differences were not statistically significant, as indicated by the overlapping confidence intervals. These findings confirm and extend previous studies that focused exclusively on mothers, ${ }^{17,} 18$ and suggest that, while maternal-specific factors cannot be fully ruled out, particularly in TD/CTD, such as the placental transmission of antibodies to the offspring - which may in turn activate the immune system in the child and alter normal brain development ${ }^{18}$ - genetic factors appear to be important. Given the increased attention to maternal immune activation in neuropsychiatric disorders, ${ }^{42,} 43$ future studies could examine if maternal infection and active AD during pregnancy are associated with increased risk of OCD and TD/CTD in their offspring and how this risk varies as a function of interaction with genetic and other environmental risk factors.

\section{Strengths and limitations}

The main strengths of this study include the uniquely large population-based cohorts of OCD and TD/CTD patients, which generally provided sufficient power to address the primary study questions, the inclusion of different kinds of biological relatives with differing degrees of shared genetic liability and shared environment, the long-term perspective (most previous studies have been cross-sectional), and the fact that the Swedish ICD codes for $\mathrm{OCD}, \mathrm{TD} / \mathrm{CTD}$, and many of the AD have been formally validated. ${ }^{25}, 29,32,33$

The results need to be interpreted in light of some limitations. First, despite the large cohorts, power was still limited for several of the individual AD, particularly streptococcusrelated diseases (such as rheumatic fever with or without chorea), which means that we could not calculate some of the specific risks or these resulted in less precise estimates (wide CI). Second, because the Swedish versions of ICD-8 and ICD-9 do not differentiate between type 1 and type 2 diabetes mellitus, we only included individuals diagnosed with type 1 diabetes mellitus according to ICD-10, resulting in the exclusion of older cases diagnosed before ICD-10 was introduced in Sweden (1997). Third, our cohorts do not represent the totality of all OCD and TD/CTD patients in Sweden; many sufferers do not seek help, the coverage of the register before 2001 is incomplete, and patients diagnosed by general practitioners and non-medical specialists are not included. In contrast, AD may be more likely to be seen by specialist physicians in Sweden, resulting in better coverage in the patient register.

\section{Clinical implications}

It may be sensible to routinely screen for and document comorbid $\mathrm{AD}$ and family history of AD in all patients with OCD and TD/CTD, particularly - but not exclusively - in those presenting with the constellation of symptoms commonly referred to as PANS or PANDAS. Long-term follow-up of these patients may provide useful clues regarding their prognosis, compared to patients without a personal or familial link to $\mathrm{AD}$. Whether patients with an $\mathrm{AD}$ link respond differently or preferentially to conventional evidence-based treatments is another interesting question for the future. As knowledge advances, it may be possible to identify robust biomarkers to help guide the clinical management of those patients. 


\section{Conclusion}

The results suggest a familial, potentially genetic link between both OCD and TD/CTD and a broad range of $\mathrm{AD}$, which is not limited to streptococcus-related conditions. At present, we cannot fully rule out shared environmental effects or additional mother-specific factors, such as the placental transmission of antibodies to their offspring, particularly in TD/CTD.

Screening for $\mathrm{AD}$ in these patients and their relatives would make good clinical sense.

\section{Supplementary Material}

Refer to Web version on PubMed Central for supplementary material.

\section{Acknowledgments}

We are grateful to Daniel S. Tylee, BA, and Stephen J. Glatt, PhD (Psychiatric Genetic Epidemiology \& Neurobiology Laboratory; Departments of Psychiatry and Behavioral Sciences \& Neuroscience and Physiology; SUNY Upstate Medical University; Syracuse, NY, U.S.A.) for sharing their unpublished data.

Conflict of interest disclosures: Prof. Mataix-Cols and Dr. Fernández de la Cruz receive royalties for contributing articles to UpToDate, Wolters Kluwer Health. Prof. Lichtenstein has served as a speaker for Medice. Prof. Leckman has received support from the NIH (salary and research), the Tourette Association of America, Grifolis, LLC, and he receives book royalties from John Wiley and Sons, McGraw-Hill, and Oxford University Press. Prof. Larsson has served as a speaker for Eli-Lilly and Shire and has received a research grant from Shire Pharmaceuticals (all outside the submitted work).

Funding/Support: The study was partly supported by a grant from the Tourette Association of America (MataixCols). Dr. Frans was supported by the Swedish Brain Foundation. Ms. Ana Pérez-Vigil was supported by a grant from the Alicia Koplowitz Foundation. Dr. Fernández de la Cruz is supported by a Junior Researcher grant from the Swedish Research Council for Health, Working Life and Welfare (FORTE grant number 2015-00569). Dr. Crowley was supported by NIMH grants R01MH105500 and R01MH110427. Dr. Rück was supported by a grant from the Swedish Research Council (K2013-61P-22168). We also acknowledge financial support from the Swedish Research Council through the $S$ wedish $I$ nitiative for Research on $M$ icrodata in the Social $A$ nd $M$ edical Sciences (SIMSAM) framework grant no 340-2013-5867. Dr. Lichtenstein is supported by grants from the Swedish Research Council for Health, Working Life and Welfare and the Swedish Research Council.

Role of the Funder/Sponsor: The funders had no role in the design and conduct of the study; collection, management, analysis, and interpretation of the data; and preparation, review, or approval of the manuscript; and decisions to submit the manuscript for publication.

\section{References}

1. Atladottir HO, Pedersen MG, Thorsen P, Mortensen PB, Deleuran B, Eaton WW, et al. Association of family history of autoimmune diseases and autism spectrum disorders. Pediatrics. 2009; 124(2): 687-694. [PubMed: 19581261]

2. Eaton WW, Byrne M, Ewald H, Mors O, Chen CY, Agerbo E, et al. Association of schizophrenia and autoimmune diseases: linkage of Danish national registers. The Am J Psychiatry 2006. 2006; 163(3):521-528.

3. Eaton WW, Pedersen MG, Nielsen PR, Mortensen PB. Autoimmune diseases, bipolar disorder, and non-affective psychosis. Bipolar Disord. 2010; 12(6):638-646. [PubMed: 20868462]

4. Swedo SE, Leonard HL, Garvey M, Mittleman B, Allen AJ, Perlmutter S, et al. Pediatric autoimmune neuropsychiatric disorders associated with streptococcal infections: Clinical description of the first 50 cases. Am J Psychiatry. 1998; 155(2):264-271. [PubMed: 9464208]

5. Macerollo A, Martino D. Pediatric Autoimmune Neuropsychiatric Disorders Associated with Streptococcal Infections (PANDAS): An Evolving Concept. Tremor Other Hyperkinet Mov (N Y). 2013; 3

6. Brown RS. Autoimmune thyroid disease: unlocking a complex puzzle. Curr Opin Pediatr. 2009; 21(4):523-528. [PubMed: 19474733] 
7. Lernmark A. Environmental factors in the etiology of type 1 diabetes, celiac disease, and narcolepsy. Pediatr Diabetes. 2016; 17 Suppl 22:65-72. [PubMed: 27411439]

8. Anaya JM. Common mechanisms of autoimmune diseases (the autoimmune tautology). Autoimmun Rev. 2012; 11(11):781-784. [PubMed: 22353402]

9. Restrepo NA, Butkiewicz M, McGrath JA, Crawford DC. Shared Genetic Etiology of Autoimmune Diseases in Patients from a Biorepository Linked to De-identified Electronic Health Records. Front Genet. 2016; 7:185. [PubMed: 27812365]

10. Cardenas-Roldan J, Rojas-Villarraga A, Anaya JM. How do autoimmune diseases cluster in families? A systematic review and meta-analysis. BMC Med. 2013; 11:73. [PubMed: 23497011]

11. Cooper GS, Bynum ML, Somers EC. Recent insights in the epidemiology of autoimmune diseases: improved prevalence estimates and understanding of clustering of diseases. J Autoimmun. 2009; 33(3-4):197-207. [PubMed: 19819109]

12. Somers EC, Thomas SL, Smeeth L, Hall AJ. Autoimmune diseases co-occurring within individuals and within families: a systematic review. Epidemiology (Cambridge, Mass). 2006; 17(2):202-217.

13. Pérez-Vigil A, Fernández de la Cruz L, Brander G, Isomura K, Gromark C, Mataix-Cols D. The link between autoimmune diseases and obsessive-compulsive and tic disorders: A systematic review. Neurosci Biobehav Rev. 2016; 71:542-562. [PubMed: 27687817]

14. de Alvarenga PG, Floresi AC, Torres AR, Hounie AG, Fossaluza V, Gentil AF, et al. Higher prevalence of obsessive-compulsive spectrum disorders in rheumatic fever. Gen Hosp Psychiatry. 2009; 31(2):178-180. [PubMed: 19269540]

15. Foroughipour M, Behdani F, Hebrani P, Marvast MN, Esmatinia F, Akhavanrezayat A. Frequency of obsessive-compulsive disorder in patients with multiple sclerosis: A cross-sectional study. J Res Med Sci. 2012; 17(3):248-253. [PubMed: 23267376]

16. Toren P, Toren A, Weizman A, Mozes T, Eldar S, Magor A, et al. Tourette's disorder: Is there an association with the antiphospholipid syndrome? Biological Psychiatry. 1994; 35(7):495-498. [PubMed: 8018803]

17. Murphy T, Storch E, Turner A, Reid J, Tan J, Lewin A. Maternal history of autoimmune disease in children presenting with tics and/or obsessive-compulsive disorder. Neuroimmunol. 2010; 229(1-2):243-247.

18. Dalsgaard S, Waltoft BL, Leckman JF, Mortensen PB. Maternal history of autoimmune disease and later development of Tourette syndrome in offspring. J Am Acad Child Adolesc Psychiatry. 2015; 54(6):495-501. [PubMed: 26004665]

19. Hounie AG, Pauls DL, do Rosario-Campos MC, Mercadante MT, Diniz JB, De Mathis MA, et al. Obsessive-compulsive spectrum disorders and rheumatic fever: a family study. Biol Psychiatry. 2007; 61(3):266-272. [PubMed: 16616727]

20. Seixas AA, Hounie AG, Fossaluza V, Curi M, Alvarenga PG, De Mathis MA, et al. Anxiety disorders and rheumatic Fever: is there an association? CNS Spectr. 2008; 13(12):1039-1046. [PubMed: 19179939]

21. Burke PM, Kocoshis S, Neigut D, Sauer J, Chandra R, Orenstein D. Maternal psychiatric disorders in pediatric inflammatory bowel disease and cystic fibrosis. Child Psychiatry Hum Dev. 1994; 25(1):45-52. [PubMed: 7805435]

22. Lee JY, Huerta PT, Zhang J, Kowal C, Bertini E, Volpe BT, et al. Neurotoxic autoantibodies mediate congenital cortical impairment of offspring in maternal lupus. Nat Med. 2009; 15(1):9196. [PubMed: 19079257]

23. Palmeira P, Quinello C, Silveira-Lessa AL, Zago CA, Carneiro-Sampaio M. IgG placental transfer in healthy and pathological pregnancies. Clin Dev Immunol 2012. 2012:985646.

24. Lunde AS, Lundeborg S, Lettenstrom GS, Thygesen L, Huebner J. The person-number systems of Sweden, Norway, Denmark, and Israel. Vital Health Stat 2. 1980; (84):1-59.

25. Rück C, Larsson KJ, Lind K, Pérez-Vigil A, Isomura K, Sariaslan A, et al. Validity and reliability of chronic tic disorder and obsessive-compulsive disorder diagnoses in the Swedish National Patient Register. BMJ open. 2015; 5(6):e007520.

26. World Health Organisation. Manual of international statistical classification of diseases, injuries, and causes of death, Vol I, 8th revision. Geneva: World Health Organisation; 1967. 
27. World Health Organisation. Manual of international statistical classification of diseases, injuries, and causes of death, Vol I, 9th revision. Geneva: World Health Organization; 1977.

28. World Health Organisation. Manual of international statistical classification of diseases, injuries, and causes of death, Vol I, 10th revision. Geneva: World Health Organization; 1993.

29. Ludvigsson JF, Andersson E, Ekbom A, Feychting M, Kim JL, Reuterwall C, et al. External review and validation of the Swedish national inpatient register. BMC public health. 2011; 11:450. [PubMed: 21658213]

30. American Autoimmune Related Diseases Association. [accessed 10 October 2015] InternetAvailable from: https://www.aarda.org/disease-list/AARDA

31. Selmi C, Barin JG, Rose NR. Current trends in autoimmunity and the nervous system. J Autoimmun. 2016; 75:20-29. [PubMed: 27545842]

32. Waldenlind K, Eriksson JK, Grewin B, Askling J. Validation of the rheumatoid arthritis diagnosis in the Swedish National Patient Register: a cohort study from Stockholm County. BMC Musculoskelet Disord. 2014; 15:432. [PubMed: 25510838]

33. Arkema EV, Jonsen A, Ronnblom L, Svenungsson E, Sjowall C, Simard JF. Case definitions in Swedish register data to identify systemic lupus erythematosus. BMJ open. 2016; 6(1):e007769.

34. Pettersson E, Larsson H, Lichtenstein P. Common psychiatric disorders share the same genetic origin: a multivariate sibling study of the Swedish population. Mol Psychiatry. 2016; 21(5):717721. [PubMed: 26303662]

35. Tylee DS, Hess JL, Tahir MA, Sharma E, Malik R, Worrall BB, et al. Genetic correlations among brain-behavioral and immune-related phenotypes based on genome-wide association data. bioRxiv. 2016:070730.

36. Stewart SE, Yu D, Scharf JM, Neale BM, Fagerness JA, Mathews CA, et al. Genome-wide association study of obsessive-compulsive disorder. Mol Psychiatry. 2013; 18(7):788-798. [PubMed: 22889921]

37. Mattheisen M, Samuels JF, Wang Y, Greenberg BD, Fyer AJ, McCracken JT, et al. Genome-wide association study in obsessive-compulsive disorder: results from the OCGAS. Mol Psychiatry. 2015; 20(3):337-344. [PubMed: 24821223]

38. Scharf JM, Yu D, Mathews CA, Neale BM, Stewart SE, Fagerness JA, et al. Genome-wide association study of Tourette's syndrome. Mol Psychiatry. 2013; 18(6):721-728. [PubMed: 22889924]

39. Roessner V, Hoekstra PJ. European Multicenter Tics in Children Studies (EMTICS): exploring the onset and course of tic disorders. Eur Child Adolesc Psychiatry. 2013; 22(7):451-452. [PubMed: 23839222]

40. Georgitsi M, Willsey AJ, Mathews CA, State M, Scharf JM, Paschou P. The Genetic Etiology of Tourette Syndrome: Large-Scale Collaborative Efforts on the Precipice of Discovery. Front Neurosci. 2016; 10:351. [PubMed: 27536211]

41. Network and Pathway Analysis Subgroup of Psychiatric Genomics Consortium. Psychiatric genome-wide association study analyses implicate neuronal, immune and histone pathways. Nat Rev Neurol. 2015; 18(2):199-209.

42. Knuesel I, Chicha L, Britschgi M, Schobel SA, Bodmer M, Hellings JA, et al. Maternal immune activation and abnormal brain development across CNS disorders. Nat Rev Neurol. 2014; 10(11): 643-660. [PubMed: 25311587]

43. Estes ML, McAllister AK. Maternal immune activation: Implications for neuropsychiatric disorders. Science (New York, NY). 2016; 353(6301):772-777. 


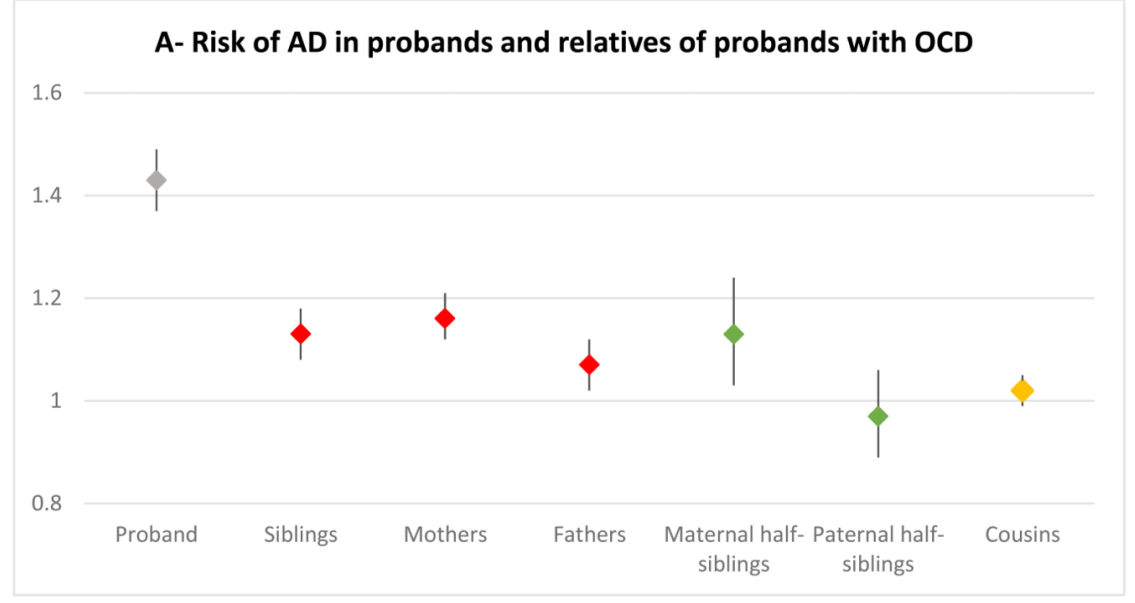

B- Risk of AD in probands and relatives of probands with TD/CTD

1.6

1.6

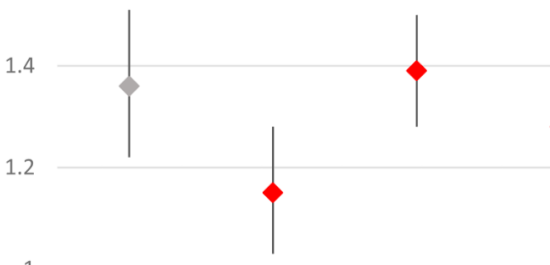

1

0.8

Proband Siblings Mothers Fathers Maternal half- Paternal half- Cousins

Figure 1.

Sensitivity analyses. Risk (ORs and 95\% CI) of any AD in probands and relatives of probands with OCD (panel A) and TD/CTD (panel B). Analyses exclude any AD amongst probands as well as OCD or TD/CTD in the relatives (full data available in Supplementary Table 5).

Footnotes: Gray shapes = probands; red shapes $=$ first-degree relatives, green shapes = second-degree relatives, yellow shapes $=$ third-degree relatives.

Abbreviations: $\mathrm{AD}=$ autoimmune diseases; $\mathrm{CI}=$ confidence interval $; \mathrm{CTD}=$ chronic tics disorder; $\mathrm{OCD}=$ obsessive-compulsive disorder; $\mathrm{OR}=$ odds ratio; $\mathrm{TD}=$ Tourette's disorder. 


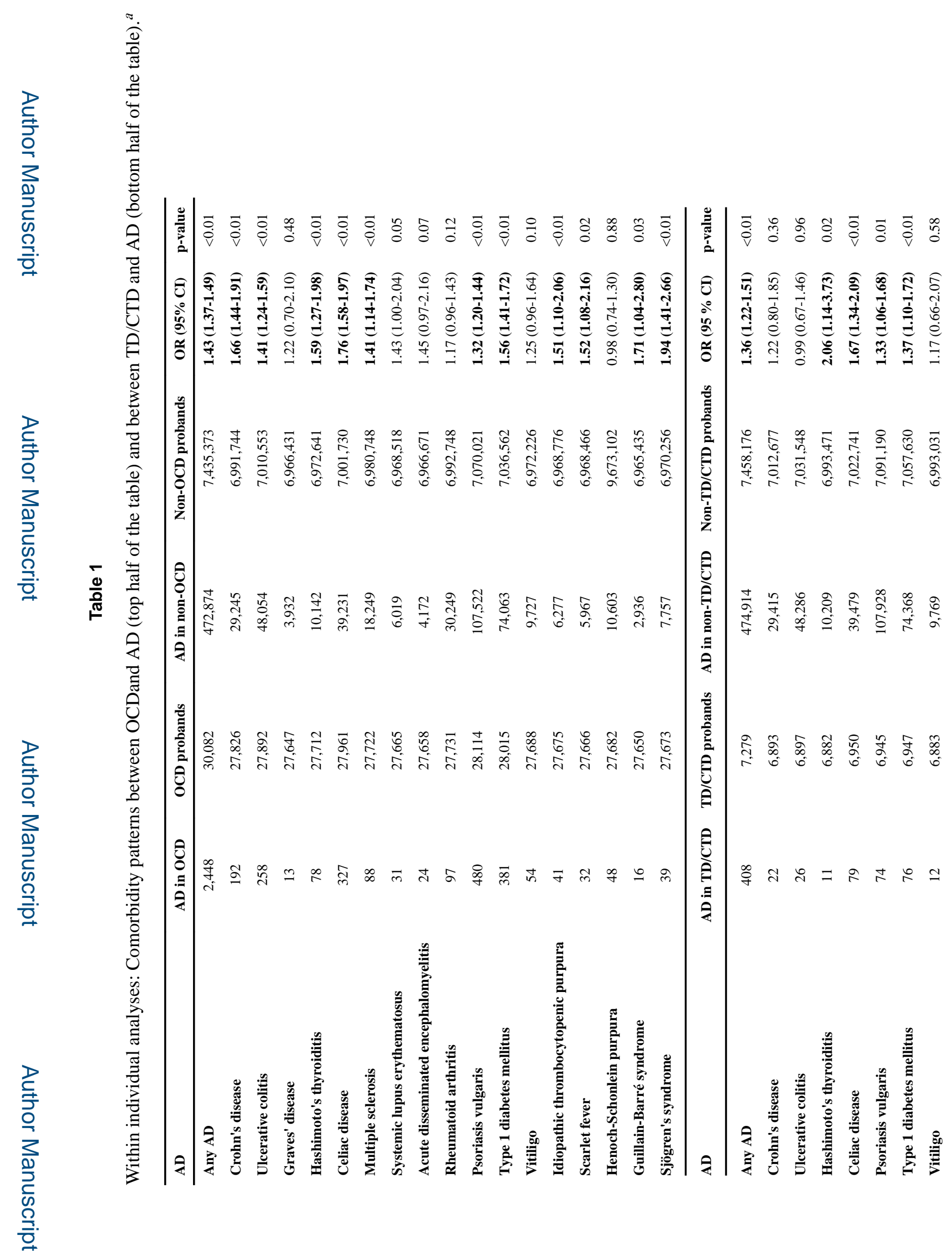

Mol Psychiatry. Author manuscript; available in PMC 2019 January 01. 


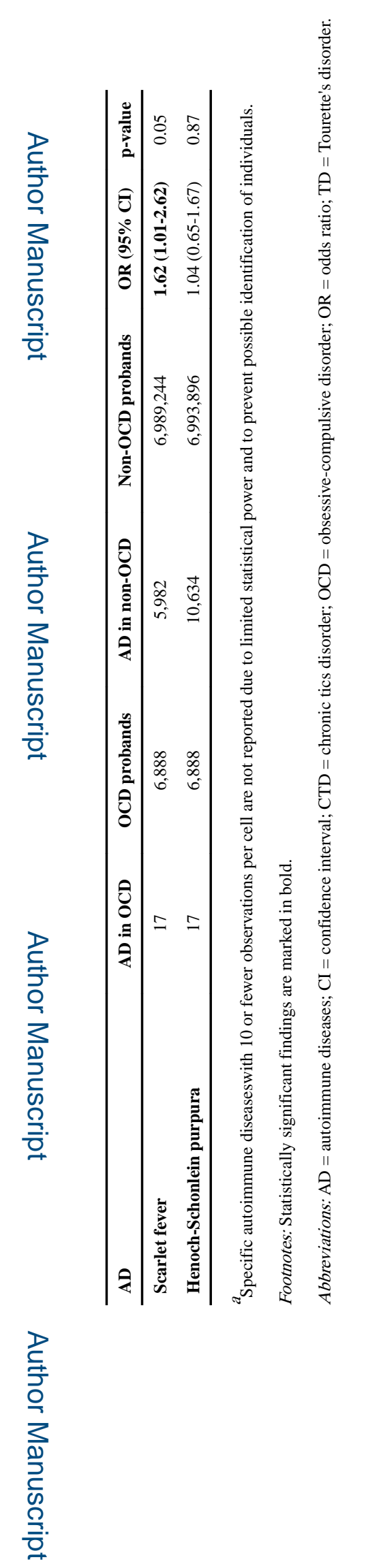

Mol Psychiatry. Author manuscript; available in PMC 2019 January 01. 


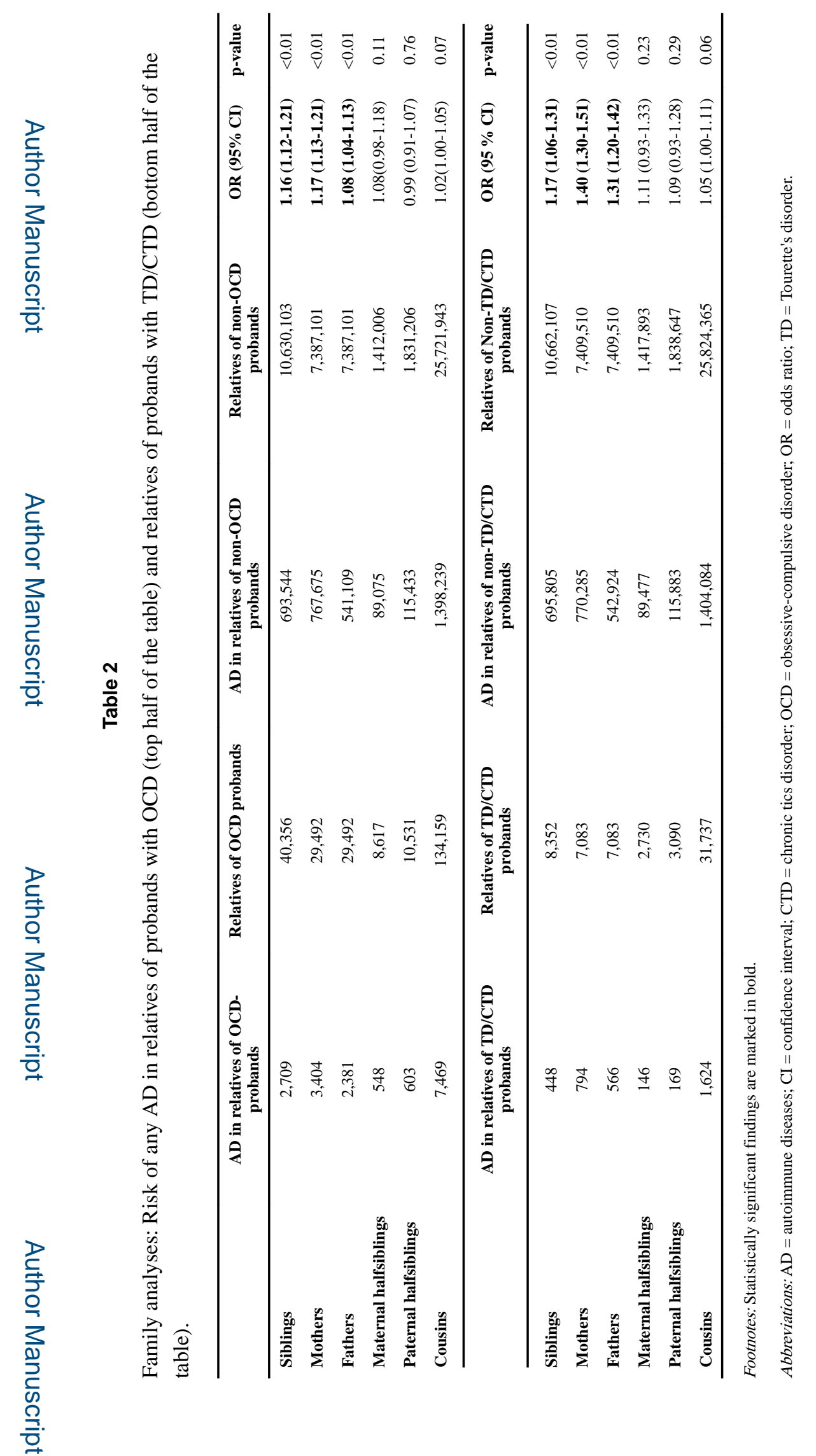

Mol Psychiatry. Author manuscript; available in PMC 2019 January 01. 